\title{
Los jóvenes de la ciudad de Popayán frente al cambio climático. Un estudio desde las representaciones sociales
}

\author{
Nilsa Lorena Alvear-Narváez \\ Victoria Eugenia Ceballos-Sarria \\ Maricela Urbano-Bolaños ${ }^{* *}$
}

Recibido: 20/01/2014 - Aceptado: 12/12/2014

\begin{abstract}
Resumen
La presente investigación se realizó en el marco del proyecto "Representaciones ante el cambio climático: conocimientos, creencias y comportamientos de la comunidad del municipio de Popayán", ejecutado por el Grupo de Estudios Ambientales (GEA) de la Universidad del Cauca. Tuvo por objeto explorar las representaciones frente al cambio climático en una población urbana, como referente para proponer criterios base en la formulación de estrategias de educación de los programas de investigación que en cambio climático desarrolla, como el de "Monitoreo Ambiental para Cambio Climático en Ecosistemas Agrícolas Altoandinos (MACACEA)". En este caso se presenta el estudio realizado a través de un instrumento demoscópico con población entre los 15 y los 29 años de edad residente en la zona urbana de Popayán, respecto a temas núcleo sobre cambio climático como son: conocimientos y creencias sobre causas, procesos y consecuencias del cambio climático, la importancia atribuida y las fuentes de información.
\end{abstract}

Palabras clave: representaciones sociales, ecosistemas urbanos, problemáticas ambientales globales, educación ambiental, gestión ambiental urbana.

* Bióloga. Especialista en Gestión Ambiental Urbana y Educación Ambiental. Estudiante del programa de Doctorado en Ciencias Ambientales de la Universidad del Cauca-Popayán, Colombia. Investigadora asociada al Grupo de investigación en Estudios Ambientales de la Universidad del Cauca. Calle 2 N. 1A-25 Urbanización Caldas.nalvear@unicauca.edu.co

** Bióloga. Especialista en Gestión Ambiental Urbana. Investigadora asociada al Grupo de investigación en Estudios Ambientales de la Universidad del Cauca. Calle 2 N. ${ }^{\circ}$ A-25 Urbanización Caldas. victoriaceballos@unicauca. edu.co

*** Ingeniera Ambiental. Pasante en el Grupo de investigación en Estudios Ambientales de la Universidad del Cauca. Calle 2 N.1A-25 Urbanización Caldas. mariurb87@gmail.com 


\title{
Young people of popayan city facing climate change. A study from the social representations
}

\begin{abstract}
This research was conducted under the project "Representations to Climate Change: Knowledge, Beliefs and Behaviors of the community in the municipality of Popayán", implemented by the Environmental Studies Group (GEA) at the University of Cauca. Its purpose was to explore the representations addressing climate change in an urban population, as a reference basis for proposing criteria in formulating strategies for education programs in climate change research that develops, such as "Environmental Monitoring for Climate Change High Andean Agricultural ecosystems (MACACEA) ". In this case study through an instrument demoscopic population between 15 and 29 years of age residing in urban area of Popayan, core issues regarding climate change is presented as: knowledge and beliefs about causes, processes and consequences of climate change, and the importance attributed sources.
\end{abstract}

Key words: social representations, urban ecosystem, global environmental issues, environmental education, urban environmental management. 


\section{INTRODUCCIÓN}

Entre las problemáticas ambientales globales actuales se encuentra el cambio climático (CC), constituido durante las últimas décadas en un tema de preocupación por su estrecha relación con el desarrollo económico y social. El informe "Perspectiva Mundial sobre la Diversidad Biológica" del 2010 [1] confirma que el CC está identificado como una de las cinco presiones globales que afectan la biodiversidad y requiere analizarse $\mathrm{y}$ enfrentarse desde una perspectiva global y local, puesto que ha surgido como consecuencia de múltiples factores interactuantes [2,3].

La información científica disponible indica que los cambios que el clima ha tenido en las últimas décadas, particularmente los aumentos de temperatura, están influyendo en los sistemas físicos y biológicos en los entornos acuáticos, terrestres y marinos. La vulnerabilidad de las sociedades humanas y de los sistemas naturales a climas extremos queda demostrada por los daños, dificultades y muertes consiguientes a sucesos tales como sequías, inundaciones, olas de calor, aludes y torbellinos [2] que se han presentado en diferentes regiones del planeta en los últimos años, un CC que debe reconocerse como problemática ambiental y social, enfrentarse de forma sistémica e involucrar tanto responsables políticos como al conjunto de la sociedad [4].

"La inconsciencia del hombre frente a la destrucción del mundo" (hombre, 23 años), "cambios bruscos de temperatura" (hombre, 29 años), "nosotros somos los responsables del cambio climático" (mujer, 24 años), "es una serie de fenómenos naturales que suceden en el planeta, algo fuera de lo normal" (mujer, 18 años), son algunas de las frases mencionadas espontáneamente por los jóvenes de la zona urbana de la ciudad de Popayán durante la presente investigación, al preguntarles qué es para ellos el CC.

González y Cruz [5] aluden al CC como una abstracción, pues las abstracciones no son fáciles de entender para muchas personas a quienes les cuesta trabajo extrapolar el significado de un concepto a un conjunto de fenómenos naturales que sacuden al mundo físico. Además, el CC está ligado a las actividades económicas, culturales, sociales y biológicas, y tiene una raíz histórica, lo que lo hace un fenómeno de gran complejidad epistemológica que desafía al conocimiento científico disciplinario. Lezama [6] señala que un problema puede tener existencia física real, pero si no es socialmente percibido y asumido como tal termina siendo socialmente irrelevante, pues son las normas, los símbolos sociales, la ideología y el poder los que le asignan a un problema su prioridad de atención. Aunque los asuntos ambientales sean identificados y evaluados en el dominio de la ciencia, es necesario que esta información se incorpore al sentido común para que sean asumidos como tales [7].

Desde sus investigaciones, Meira [8] concluye que la sociedad tiene un papel creativo a la hora de integrar la información, de interpretarla y usarla para darle sen- 
tido a la realidad; por ello es importante partir de las representaciones sociales (RS) para definir acciones adecuadas a la realidad local. Moscovici [9] define las RS como: “(...) una modalidad particular del conocimiento, cuya función es la elaboración de los comportamientos y la comunicación entre los individuos... La representación es un corpus organizado de conocimientos y una de las actividades psíquicas gracias a las cuales los hombres hacen inteligible la realidad fisica y social, se integran en un grupo o en una relación cotidiana de intercambios, liberan los poderes de su imaginación".

Meira [10] resalta la necesidad de explorar las RS del CC por dos cuestiones. Primero, porque es una variable susceptible de ser manipulada para obstruir cualquier alternativa que amenace determinados intereses en juego. Segundo, puede ser instrumentalizada como un factor social positivo en la medida en que cualquier acción estructural sobre las causas últimas del CC debe contar con la comprensión y el consenso ciudadano, máxime si implica cambios significativos en los estilos de vida y los patrones de bienestar aceptados en las sociedades avanzadas y deseados por prácticamente todo el mundo.

La educación ambiental se presenta como una estrategia fundamental para la formación de ciudadanía con la que afrontar el CC. Es así y bajo este contexto, que desde los estudios de RS se pretende aportar a la educación ambiental bajo condiciones particulares de CC local. La educación ambiental no producirá impacto si no tiene un enfoque contextual, conceptual y metodológico adecuado y pertinente [11]. Ciertamente se demanda de su ambientación, puesto que se requieren principios y categorías que ayuden a diferenciar la situación real, ya que cada visión se acerca y propone acciones diferentes con alcances distintos [12].

El fenómeno del CC ha cobrado una relevancia inusitada en el ámbito internacional. $\mathrm{Su}$ emergencia ha reactivado la política ambiental en su conjunto y, en particular, la educación ambiental, y por ello se ha incrementado su presencia en tratados internacionales, entre ellos el de la Convención Marco de las Naciones Unidas sobre Cambio Climático (CMNUCC) que, en su artículo 6, destaca la importancia de estrategias de educación, formación y sensibilización como fundamentales para mejorar la apropiación del conocimientos acerca del CC. Colombia, como miembro de la CMNUCC, publicó en 2010 la "Estrategia Nacional de Educación, Formación y Sensibilización de Públicos sobre Cambio Climático" [13] en donde se llama a la necesidad de implementar acciones de cumplimiento no solo a partir de la inserción de la estrategia en la planificación sectorial e institucional, sino también a través de la implementación de programas y proyectos de adaptación y mitigación al CC con participación ciudadana, ajustados a las necesidades, realidades y capacidades locales. 
El Grupo de Investigación en Estudios Ambientales (GEA) de la Universidad del Cauca en los últimos años ha desarrollado programas de investigación en el tema de CC en ecosistemas urbanos y agrícolas altoandinos [14, 15], programas en los cuales se ha considerado que es fundamental un componente de educación ambiental con el objeto de formular criterios y/o estrategias educativas integrales con los actores involucrados, que aporten a los procesos locales de adaptación al CC a partir de una apropiación social del conocimiento, y que sirvan de base a los actores en la toma de decisiones sobre acciones frente al CC y la sostenibilidad de la región. En este marco surgió la investigación "Representaciones sociales ante el cambio climático: conocimientos, creencias y comportamientos de la comunidad urbana", que tiene como objeto indagar sobre las RS que tiene la población residente en la zona urbana de Popayán ante el CC, para determinar cómo sus conocimientos y creencias condicionan sus comportamientos cotidianos y sus acciones ante las políticas nacionales y que la información sirva de base para procesos socioeducativos. El estudio se realizó en el total de la población urbana de la ciudad, pero en la presente investigación se muestra solamente la información sobre la población joven comprendida entre los 15 y los 29 años de edad.

La investigación se desarrolló en el municipio de Popayán, ubicado al suroccidente de Colombia en el departamento del Cauca. De acuerdo con el censo nacional del 2005 [16], se reportan 257512 habitantes, el $88 \%$ asentados en la zona urbana, predominando la población con edades de 15 a 44 años. Entre los grupos étnicos asentados en la zona urbana están: el cabildo urbano de la etnia Yanaconas, los Coconucos y los afrocolombianos. La zona urbana del municipio se divide en nueve comunas, constituidas por 282 barrios aproximadamente. Los ecosistemas naturales se encuentran en las zonas de vida de bosque andino y subandino. Sus principales sectores económicos son: agrícola, pecuario y ambiental [17].

\section{MATERIALES Y MÉTODOS}

La metodología empleada atiende a aspectos relacionados con el CC y con la teoría de las representaciones sociales, además de los aportes conceptuales y metodológicos que sobre representaciones sociales frente al cambio climático se están desarrollando en los grupos de investigación de González y Meira en diversos estudios de Latinoamérica y España [7], las cuales se adaptaron para el presente caso de estudio, sin desconocer que existen otros procesos metodológicos que abordan el tema.

La investigación se realizó con jóvenes entre los 15 y los 29 años residentes en la zona urbana de Popayán. Se realizó con el fin de indagar las representaciones sociales en el segmento de los jóvenes de la zona urbana, aportando información del imaginario social y la dimensión simbólica para identificar representaciones y percepciones 
sociales de este grupo poblacional que frente al cambio climático han construido. El método que se empleó fue un instrumento demoscópico integrado por 12 preguntas entre cerradas, de opción múltiple, y abiertas. Se hicieron 5 preguntas adicionales para conocer el género, la edad, estrato socioeconomico, el nivel de estudios y la ocupacion de los encuestados. La información resultante fue capturada en una base de datos empleando el programa informático SPSS para su manejo estadístico. Las preguntas abiertas fueron agrupadas en conceptos integradores de respuesta para incorporarlos a la base de datos general, y hacer un análisis general descriptivo.

El instrumento se aplicó en los meses de julio y agosto de 2011. Para la definición de la muestra se utilizó la metodología de diseño muestreal no probabilístico por cuotas, según las condiciones de género y edad, tomando como referente la información del censo DANE [16]. Por tanto, la muestra estuvo comprendida por 98 personas. Para el diseño de la encuesta se tuvieron en cuenta las tres dimensiones de las representaciones sociales establecidas por Moscovici [9] y para el análisis de la encuesta se tuvieron en cuenta tres ejes enfocados a (i) conocimientos y creencias del proceso del $\mathrm{CC}$, (ii) importancia que le atribuye la población como problemática global y local, y (iii) fuentes de información.

\section{RESULTADOS Y DISCUSIÓN}

En género, el porcentaje de mujeres encuestadas fue de $53 \%$ y $47 \%$ de hombres. El estrato socioeconómico más representado por la población encuestada fue el 3 con el $45 \%$, seguido del 4 con $20 \%$, el 2 con el $19 \%$ y los estratos 1,5 y 6 con menos del $8 \%$. En nivel de estudios, el mayor porcentaje lo registran las personas con estudios de pregrado con un $50 \%$, seguido de los que cuentan con estudios secundarios con el $46 \%$ y los de estudios de posgrado, sin estudios y primarios con menos del $3 \%$. Con relación a la ocupación, la mayoría la representan estudiantes con $69 \%$, trabajadores dependientes con $16 \%$ y con valores porcentuales inferiores al $6 \%$ se reportan los trabajadores independientes, desempleados y en otra situación.

\subsection{El cambio climático atribuido como problemática ambiental global y local}

Para conocer si los jóvenes de Popayán identifican al CC como una problemática presente, se indagó con preguntas abiertas sobre los problemas que consideran relevantes en el mundo, en Colombia y en Popayán. Para el análisis de las preguntas abiertas se agruparon las respuestas en conceptos integradores.

En la escala mundial los problemas mencionados son, calentamiento global y cambio climático con el $23 \%$ y el $17 \%$ respectivamente, porcentajes superados por los entrevistados que comentaron no tener una opinión al respecto con un $31 \%$. En lo 
nacional, se destacan problemas de tipo sociopolítico como conflicto armado, violencia, narcotráfico, corrupción, desempleo, mientras que el CC solo reportó un $3 \%$ y $37 \%$ de los entrevistados dijeron no opinar. Entre los problemas enunciados para Popayán, prevalecen también los sociopolíticos como desempleo, delincuencia, inseguridad, corrupción, dejando al CC mencionado solo por el $3 \%$ de los encuestados, y se resalta un porcentaje del $52 \%$ que respondió no saber qué problemas afectan a su ciudad.

Adicionalmente se preguntó a los jóvenes sobre la importancia que se le está dando al CC en el orden nacional y departamental; en los dos casos, el $86 \%$ y el $90 \%$ respectivamente, de los entrevistados manifiestan que al CC no se le está dando la importancia que tiene.

Al preguntar, quiénes serán los más afectados por el CC, el $53 \%$ opina que afectará a las generaciones actuales y futuras, y para el $77 \%$ afectará a comunidades urbanas y rurales de igual manera.

Lo anterior refleja que la población joven reconoce el término "cambio climático", siendo comprensible al tratarse de un tema que cada vez es más nombrado en medios masivos, cumbres mundiales y grupos sociales. Sin embargo, conocer el término no significa reconocerlo como una de las principales problemáticas ambientales de su contexto inmediato y lejano, aunque sí reconocen su presencia por los impactos que generaría en las generaciones actuales y futuras, y en los contextos urbanos y rurales, no es relevante para el ambiente, como sí es, por ejemplo, la contaminación. El CC no es solo un problema ambiental, pues también tiene incidencia en otros sistemas como el político y el económico, así que, aunque una problemática como el cambio climático tenga existencia física, si no es socialmente percibida y asumida como tal, termina siendo socialmente irrelevante [6].

\subsection{Fuentes de información}

En la pregunta relacionada con los medios de comunicación a través de los cuales los jóvenes de Popayán reciben información sobre $\mathrm{CC}$, los mayores porcentajes de afirmación los obtuvieron: Internet $95 \%$; televisión $93 \%$, y las clases académicas, $79 \%$ seguidos por los periódicos $68 \%$; las campañas publicitarias $66 \%$, y la radio $63 \%$; en último lugar de afirmación, se encuentran los libros $48 \%$, y revistas especializadas $54 \%$.

El CC es un tema cada vez más mencionado en los medios de comunicación, principalmente asociándolo a la ocurrencia de fenómenos climáticos extremos y sus efectos relacionados en la población y la economía, lo que hace que se muestre su relación con sucesos catastróficos y que se transmitan elementos conceptuales confusos, lo que concuerda con el eje anteriormente analizado en que los jóvenes evidencian princi- 
palmente los impactos físicos del CC. Al tratarse de población joven es comprensible que sean Internet, la TV y las clases académicas los medios por los cuales más reciben información. Además, se identifica que los dos primeros son los medios que se deben tener en cuenta en las estrategias de comunicación educativa del CC.

\subsection{Conocimientos acerca de las causas y consecuencias del cambio climático}

En la pregunta que indaga acerca de las afirmaciones relacionadas con las causas del $\mathrm{CC}$, se encontró que el $71 \%$ de los entrevistados consideran que es un proceso causado por el hombre; el $23 \%$ lo atribuye a una combinación de causas naturales y de acciones del hombre, y solo el $5 \%$ cree que el CC es un proceso natural de la tierra (figura 1), acercándose acertadamente a las definiciones que de CC se presentan por las comunidades científicas.

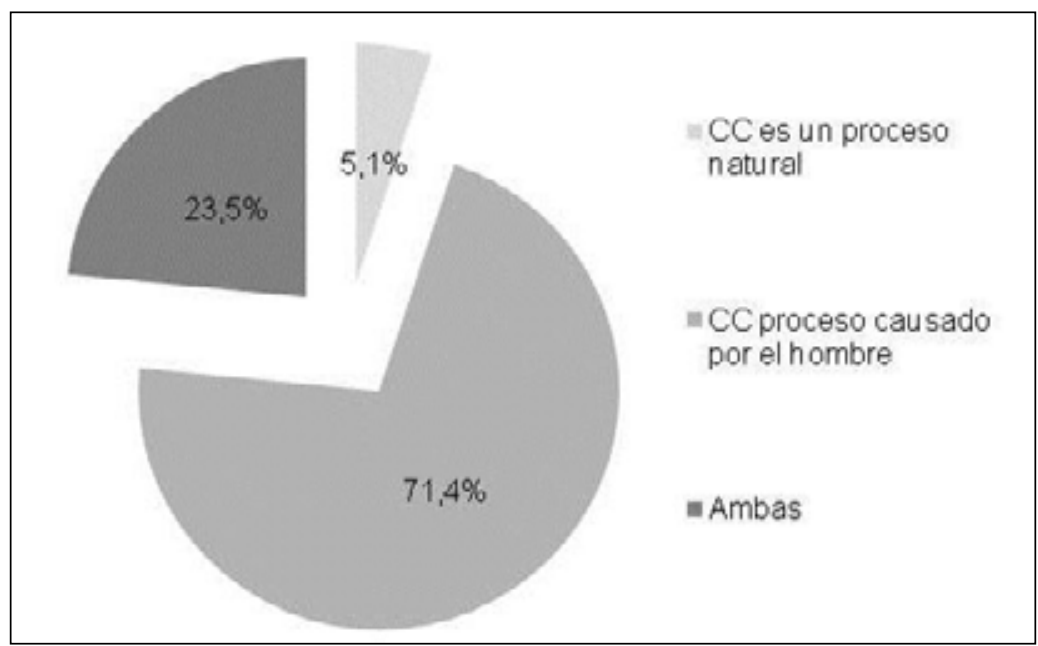

Figura 1. ¿Qué es cambio climático para los jóvenes de Popayán?

Fuente: elaboración propia

Para evaluar sobre cuáles serían las causas del CC y las acciones que contribuyen a causarlo, se plantearon interrogantes en donde los jóvenes debían responder de forma afirmativa o negativa a cada oración (tabla 1). Para el $59 \%$ de los encuestados "el CC está causado principalmente por la emisión de gases efecto invernadero". En la afirmaciones "el CC está causado por el agujero en la capa de ozono" y el "CC está causado por las lluvias ácidas", se encontró que el $31 \%$ y el 17 \%, respectivamente, de los entrevistados las considera ciertas, siendo que el agujero en la capa de ozono y las lluvias ácidas no son causas determinantes del CC. 
Tabla 1. Opiniones de causas del cambio climático

\begin{tabular}{|l|c|c|}
\hline \multicolumn{1}{|c|}{ Afirmaciones } & Si (\%) & No (\%) \\
\hline El CC está causado por un agujero en la capa de ozono & 31 & 69 \\
\hline El CC está causado principalmente por la emisión de gases efecto invernadero & 59 & 41 \\
\hline El CC está causado por las lluvias ácidas & 17 & 83 \\
\hline Contribuimos a empeorar el CC al usar petróleo, carbón y gas & 51 & 49 \\
\hline Contribuimos a empeorar el CC cuando talamos bosques & 59 & 41 \\
\hline El mal manejo de los residuos sólidos contribuye a empeorar el CC & 56 & 44 \\
\hline
\end{tabular}

Fuente: elaboración propia

Asimismo, en un estudio realizado en el año 2013 con aproximadamente 1000 estadounidenses mayores de 18 años [19], se encontró que son menos quienes defienden el punto de vista de los escépticos del calentamiento global que el aumento a largo plazo de la temperatura media de la Tierra es el resultado de los cambios naturales en el medio ambiente, y no a causa de las actividades humanas. E1 57 \% de los estadounidenses dijo que para el 2013 el calentamiento global es causado por las actividades humanas, por encima del $50 \%$ en 2010.

En cuanto a las acciones que contribuyen a causar el CC, en la afirmación "contribuimos a empeorar el cambio climático cuando usamos petróleo, carbón y gas", el $51 \%$ de los jóvenes de Popayán la consideran verdadera; al igual que las frases "contribuimos a empeorar el cambio climático cuando talamos bosques" y "el mal manejo de los residuos sólidos contribuye a empeorar el cambio climático" con un 59 \% y un $56 \%$, respectivamente.

En otra pregunta se indagó acerca de las responsabilidades que tienen diferentes actores sociales e institucionales en causar el CC. Los encuestados indicaron que las grandes industrias tienen la responsabilidad máxima en causarlo con el $97 \%$; seguido de los ciudadanos con $85 \%$, y los gobiernos internacionales $84 \%$ (tabla 2 ). Actividades como la ganadería y la agricultura que tienen un gran aporte al CC no son consideradas por los jóvenes como una causa.

Tabla 2. Responsabilidad (R.) en causar el cambio climático

\begin{tabular}{|l|c|c|c|}
\hline \multicolumn{1}{|c|}{ Actores } & R. mínima (\%) & R. máxima (\%) & No sabe (\%) \\
\hline Las grandes industrias & 3 & 97 & 0 \\
\hline Los agricultores y ganaderos & 70 & 28 & 2 \\
\hline
\end{tabular}


52 Nilsa Lorena Alvear-Narváez • Victoria Eugenia Ceballos-Sarria • Maricela Urbano-Bolaños

\begin{tabular}{|l|c|c|c|}
\hline \multicolumn{1}{|c|}{ Actores } & R. mínima (\%) & R. máxima (\%) & No sabe (\%) \\
\hline Los ciudadanos & 15 & 85 & 0 \\
\hline Los científicos & 47 & 46 & 7 \\
\hline Los medios de comunicación & 65 & 33 & 2 \\
\hline El gobierno nacional & 18 & 80 & 2 \\
\hline Los gobiernos internacionales & 14 & 84 & 2 \\
\hline
\end{tabular}

Fuente: elaboración propia

En relación con las causas del CC, se preguntó a los jóvenes acerca de la probabilidad de que algunas consecuencias del CC ocurriesen en los próximos 10 años en Colombia. Para 8 de cada 10 entrevistados, las consecuencias más probables son: aumento en la temperatura y aumento en los precios de los alimentos (tabla 3); para 7 de cada 10 las consecuencias con mayor probabilidad de ocurrir son: períodos de sequía más frecuentemente, aumento de los incendios forestales, aumento de enfermedades, aumento en los precios de los servicios públicos, tendrán que usar más la bicicleta o el transporte público, y que habrá cortes del servicio de agua con mayor frecuencia.

Tabla 3. Opinión sobre ocurrencia de consecuencias del cambio climático

\begin{tabular}{|l|c|c|c|c|}
\hline \multicolumn{1}{|c|}{ Consecuencias } & $\begin{array}{c}\text { Muy } \\
\text { probable (\%) }\end{array}$ & $\begin{array}{c}\text { Algo probable } \\
\text { (\%) }\end{array}$ & $\begin{array}{c}\text { Nada } \\
\text { probable (\%) }\end{array}$ & $\begin{array}{c}\text { No sabe } \\
\text { (\%) }\end{array}$ \\
\hline Aumento de temperatura & 83 & 15 & 0 & 2 \\
\hline Periodos de sequía más frecuentes & 79 & 20 & 0 & 1 \\
\hline Aumento de los incendios forestales & 74 & 24 & 1 & 1 \\
\hline Lluvias más fuertes & 48 & 44 & 8 & 0 \\
\hline Pérdida de zonas costeras por subida del mar & 45 & 39 & 7 & 9 \\
\hline Desaparición de plantas y animales & 60 & 32 & 7 & 1 \\
\hline Aumento de enfermedades & 74 & 22 & 2 & 2 \\
\hline Aumento en precios de los alimentos & 83 & 15 & 2 & 0 \\
\hline Aumento en precios de los servicios públicos & 75 & 24 & 1 & 0 \\
\hline Desaparición de las zonas de nieve & 64 & 27 & 5 & 4 \\
\hline Subida del precio de la gasolina & 60 & 33 & 2 & 5 \\
\hline Menos variedad de alimento para comprar & 65 & 26 & 9 & 0 \\
\hline Usar más bicicleta o transporte público & 72 & 16 & 9 & 2 \\
\hline Cortes del servicio agua con mayor frecuencia & 80 & 18 & 2 & 0 \\
\hline
\end{tabular}

Fuente: elaboración propia 
Al indagar si los jóvenes de la ciudad estaban de acuerdo con la frase "el cambio climático, lo entiendo bien, sus causas y consecuencias", el $63 \%$ respondió estar de acuerdo y el $19 \%$ manifestó estar en desacuerdo (figura 2).

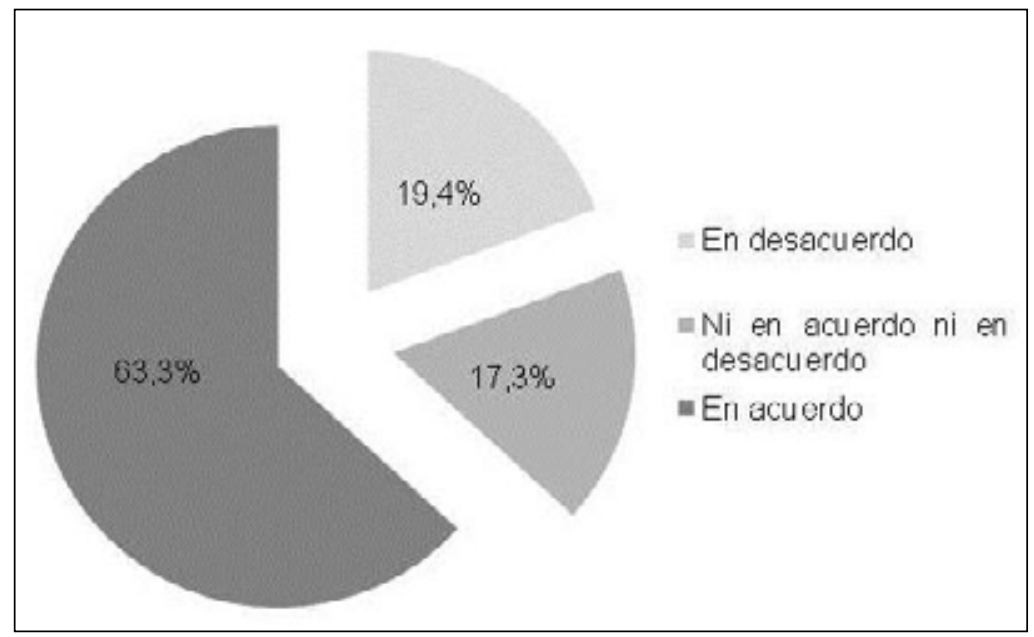

Figura 2. Los jóvenes responden a la afirmación:

"el cambio climático, lo entiendo bien, sus causas y consecuencias"

Fuente: elaboración propia

Adicionalmente se preguntó a los jóvenes encuestados acerca de las posibles consecuencias en la salud humana, y se observó que el mayor porcentaje se relaciona con problemas por el excesivo calor, con $98 \%$, seguido por más probabilidades de sufrir infecciones por la calidad del agua o de los alimentos, $97 \%$, y del riesgo de más probabilidades de tener cáncer de piel, 95\% (tabla 4).

Tabla 4. Impactos del cambio climático en la salud humana

\begin{tabular}{|l|c|c|c|}
\hline \multicolumn{1}{|c|}{ Impactos } & Si (\%) & No (\%) & $\begin{array}{c}\text { No } \\
\text { Sabe } \\
(\%)\end{array}$ \\
\hline Tendré más probabilidades de tener cáncer de piel & 95 & 2 & 3 \\
\hline Tendré más problemas con el excesivo calor & 98 & 0 & 2 \\
\hline Tendré más problemas con el excesivo frío & 66 & 27 & 7 \\
\hline Tendré más probabilidades de padecer enfermedades respiratorias & 88 & 8 & 4 \\
\hline Tendré más probabilidades de padecer enfermedades como malaria y dengue & 87 & 6 & 7 \\
\hline $\begin{array}{l}\text { Tendré más probabilidades de sufrir infecciones por la calidad del agua o } \\
\text { de los alimentos }\end{array}$ & 97 & 2 & 1 \\
\hline
\end{tabular}

Fuente: elaboración propia 


\section{CONCLUSIONES}

Entre los jóvenes de la zona urbana del municipio de Popayán se puede evidenciar que si bien entienden el proceso del CC, sus causas y consecuencias, al recibir continuamente información principalmente desde los medios masivos de comunicación como Internet y $\mathrm{TV}$, lo identifican más como una problemática global que local, puesto que en lo local son más representativos para ellos la contaminación y otros diversos problemas sociales.

Es necesario diseñar estrategias de comunicación y educación ambiental orientadas a una participación activa de la población en los procesos de adaptación y mitigación al $\mathrm{CC}$, y basados en la idea de lograr cambios de actitudes y de comportamientos sociales para contribuir a frenar el cambio climático.

La población joven debe considerarse prioritaria dentro de la sociedad colombiana y municipal por su incidencia inmediata en el ámbito político, socioeconómico y cultural, por lo cual es conveniente conocer sus representaciones y percepciones sociales de las problemáticas ambientales y con base en ellas diseñar estrategias de comunicación y educación orientadas a su participación activa en las dinámicas de adaptación y mitigación del cambio climático.

Se confirma que la educación y la comunicación del cambio climático se están reduciendo a una transmisión de información científica, especialmente a través de los medios de comunicación, pero es necesario que, por el contrario, se consoliden procesos de comunicación educativa para la formación ciudadana y la promoción de comportamientos pro-ambientales.

Los estudios de representaciones sociales, en este caso frente al cambio climático, ofrecen información de gran valor en el diseño y establecimiento de programas de comunicación educativa hacia segmentos particulares de la población, además de su potencial para impulsar medidas de participación socio-ambiental tendentes a involucrar a la juventud en la formación de su propia ciudadanía.

\section{AGRADECIMIENTOS}

Agradecimientos a los ángeles del cielo y a la naturaleza; al GEA de la Universidad del Cauca; a los encuestadores voluntarios del Colegio Campestre Americano y demás estudiantes y profesores. Finalmente a la encuestados de la ciudad de Popayán. 


\section{REFERENCIAS}

[1] Secretaría del Convenio sobre la Diversidad Biológica, "Perspectiva mundial sobre la diversidad biológica 3", 2010.

[2] Panel intergubernamental de expertos sobre el cambio climático IPCC, "Tercer informe de evaluación. Cambio Climático: impactos, adaptación y vulnerabilidad”, [En línea], Disponible: http://www.ipcc.ch/pdf/climate-changes-2001/impact-adaptation-vulnerability/impact-spmts-sp.pdf, 2001

[3] A.M. Cachá, "Estrategia local frente al cambio climático del Municipio de Murcia (Proyecto)", Agencia local de la energía y cambio climático ayuntamiento de Murcia, 2008

[4] E. Moyano y R. Lafuente, “Opinión pública y cambio climático. Ecobarómetro de Andalucía”, Instituto de estudios sociales avanzados [En línea], Disponible: http://www.inetcordoba.org/ Documentos/Publicaciones/Colaboraciones/Encuentro_analistas_08/ComSem4_1_Regina_Lafuente_Fernandez_c.pdf

[5] E. González y G. Cruz. "Estudiantes de la Universidad Veracruzana ante el cambio climático", Revista de la Universidad Veracruzana. La Palabra y el hombre. Tercera época, N. ${ }^{\circ} 23$, pp. 31- 36, 2012-2013

[6] J. Lezama, La construcción social y política del medio ambiente. México D. F.: El Colegio de México, 2008.

[7] E. González y A. Maldonado. Los jóvenes universitarios y el cambio climático: un estudio de representaciones sociales. México: Universidad Veracruzana, 2013.

[8] P. Meira. La sociedad ante el cambio climático. Conocimientos, valoraciones y comportamientos en la sociedad española. Madrid: Fundación Mapfre, 2011

[9] S. Moscovici. El psicoanálisis, su imagen y su público. Buenos Aires: Huemal, 1979

[10] P. Meira. "Las ideas de la gente sobre cambio climático", Cuadernos de Comunicación, Interpretación y Educación Ambiental, Vol. 18, pp. 5-12, 2006

[11] R. Calixto, "Medio ambiente, ciudad y género. Percepciones ambientales de educadoras", [En línea], Journal Tiempo de Educar, vol. 5, N. 9, Disponible: http://www.redalyc.org/articulo. $\underline{\text { oa? id }=31100903}, 2004$

[12] C. N. Martínez, "Percepción ambiental de los estudiantes de la Universidad Veracruzana", presentado en XI Congreso Nacional de Investigación Educativa, San Nicolás de los Garza, Nuevo León, 2011

[13] Ministerio de Ambiente, Vivienda y Desarrollo Territorial, Instituto de Hidrología, Meteorología y Estudios Ambientales de Colombia, "Estrategia nacional de educación, formación y sensibilización de públicos sobre cambio climático: segunda comunicación de Colombia ante la Convención Marco de las Naciones Unidas sobre cambio climático", 2010 
[14] A. Figueroa Casas et al., "Modelamiento climático, patrones de cambio y sus efectos en ecosistemas agrícolas altoandinos, identificando medidas de adaptación y mitigación para la planificación MACACEA”, Ministerio de Agricultura y Desarrollo Rural 84, 2012

[15] GEA, G., CIAgua y CDKN, "Interinstitucional, multi-sectorial analysis of vulnerability and adaptation to climate change for the agricultural sector in the upper Cauca river basin impacting adaptation policies", GEA, 2013

[16] Departamento administrativo nacional de estadística, "Censo general 2005. Nivel nacional”, [En línea], acceso 17 de mayo, 2010; Disponible: http://www.dane.gov.co/censo/files/libroCenso2005nacional.pdf, 2005

[17] Municipio de Popayán. Plan de Ordenamiento Territorial de Popayán. Documento técnico: Capitulo I. Dimensión Ambiental, 2002

[18] P. Meira, et al., "La sociedad ante el cambio climático. Conocimientos, valoraciones y comportamientos en la población española", Fundación MAPFRE, 2009

[19] L. Saad, “American〉s concerns about global warming on the rise”, [En línea], acceso 13 de junio, 2013; Disponible: http://www.gallup.com/poll/161645/americans-concerns-globalwarming-rise.aspx. 